\title{
Fatores associados à prevalência de diabetes auto-referido entre idosos de Minas Gerais
}

\author{
Ana Paula Franco Viegas-Pereira* \\ Roberto Nascimento Rodrigues** \\ Carla Jorge Machado***
}

\begin{abstract}
A prevalência de diabetes vem aumentando mundialmente e, neste artigo, foram analisados alguns de seus fatores associados, focalizando o segmento de idosos residentes em Minas Gerais. Foi utilizado o método de estudo seccional, com dados da PNAD-2003, envolvendo amostra de 3.662 pessoas com 60 anos e mais. Os fatores associados foram organizados segundo os níveis individual e domiciliar/comunitário, e analisados por meio de cinco modelos de regressão logística multivariada. O primeiro modelo incluiu apenas variáveis mais distantes; as demais foram incluídas sucessivamente, de acordo com sua proximidade ao diabetes. No modelo final, a chance de ter diabetes foi maior para: os idosos residentes na área urbana em relação à rural; as mulheres versus homens; os hipertensos em relação aos não hipertensos; aqueles com doença do coração em relação aos sem doença do coração; pensionistas versus não pensionistas; sem instrução e com 1 a 3 anos de estudo em comparação aos com cinco anos ou mais de estudo. A chance foi menor entre aqueles em domicílios unipessoais versus os de mais de uma pessoa, com 70 a 74 anos versus 60 a 64 anos, com renda inferior a um salário mínimo e mais de 3 até 5 salários mínimos em relação àqueles com cinco e mais salários mínimos. A relação entre renda individual do idoso e diabetes indica a necessidade de estudos que investiguem o acesso dos idosos aos serviços de saúde e que influenciam a auto-referência à morbidade.
\end{abstract}

Palavras-chave: Fatores associados. Diabetes. Diabetes mellitus. Diabetes mellitus tipo 2.

\section{Introdução}

Em 2002, cerca de 173 milhões de pessoas na idade adulta, no mundo, tinham diabetes (WHO, 2003). No Brasil, a estimativa é de que dez milhões de pessoas tenham essa doença e cerca de $90 \%$ desses indivíduos sejam portadores do diabetes tipo 2, um tipo de diabetes mellitus não insulino-dependen- te, cujo aumento tem sido observado desde a última década (OLIVEIRA; MILECH, 2004). Essas estatísticas evidenciam e justificam que o diabetes seja considerado, cada vez mais, um problema de saúde pública a ser cotejado na organização dos serviços de saúde e nas suas estratégias de atuação. $\mathrm{O}$ interesse nas ações direcionadas à prevenção e ao tratamento do diabetes ganha

\footnotetext{
* Doutora em Demografia, gestora de análise da informação estratégica na Unimed.

** Doutor em Demografia, professor titular da UFMG.

*** Doutora em Dinâmica de População, professora adjunta da UFMG.
} 
destaque, sobretudo, quando se avolumam as discussões sobre a qualidade de vida e expectativa de vida saudável.

Estudos recentes têm demonstrado que, contrariamente ao que se pensava, a incidência do diabetes tem apresentado tendência de crescimento não apenas nos países desenvolvidos, mas também naqueles em desenvolvimento (WHO, 2003). No Brasil, o aumento da prevalência do diabetes tem sido atribuído a transformações demográficas, como o envelhecimento populacional, e aos processos de industrialização e urbanização. Entre os idosos, no Brasil, a prevalência de diabetes é elevada, acometendo $17,3 \%$ das pessoas com 60 a 69 anos (LOURENÇO, 2004). Com isso, há elevação no número de consultas, solicitações de exames, internações e cirurgias, que resultam, muitas vezes, em incapacitação laborativa provisória ou permanente (OLIVEIRA; MILECH, 2004). Em projeção de carga de doença no Brasil, Leite et al. (2005) destacam que, tanto em 1998 quanto em 2013, o diabetes mellitus constitui a causa específica com maior participação nos anos de vida perdidos ajustados por incapacidade, para ambos os sexos.

No entanto, aspectos sociodemográficos e de saúde de idosos com diabetes no Brasil têm sido pouco focalizados, em parte porque as bases de dados adequadas para esse tipo de estudo são escassas. Mas, a partir dos anos 90 , têm surgido pesquisas de cobertura nacional sobre morbidade por causas no Brasil, a mais recente delas propiciada pela PNAD 2003. Isto possibilita o empreendimento de análises sobre diferentes dimensões ou aspectos sociodemográficos da prevalência de diabetes no Brasil. Este é o foco do presente estudo, que se refere particularmente ao universo amostral constituído pelo Estado de Minas Gerais.

Assim, o objetivo deste trabalho é analisar fatores associados à prevalência de diabetes auto-referido entre idosos de 60 anos ou mais, residentes no Estado de Minas Gerais, em 2003, considerando-se dois níveis de exposição aos fatores de risco ou de vulnerabilidade: individual e domiciliar/comunitário.
Apesar do surgimento de técnicas diagnósticas, imunização e terapêuticas específicas e apuradas, ainda não foi possível obter uma explicação completa sobre a incidência de diabetes. Isto ressalta a necessidade de uma abordagem holística da complexidade do processo saúde-doença que considere os distintos níveis de exposição aos fatores de risco, ou de vulnerabilidade, relacionados tanto às condições de vida e do meio ambiente quanto ao estilo de vida. Para isso, uma possibilidade é agregar os fatores associados em quatro grupos (BLOCH, 1998): características constitucionais do indivíduo (inerentes e não passíveis de modificações); características comportamentais determinadas pelo ambiente psico-socioeconômico; patologias ou distúrbios metabólicos; e características socioeconômico-culturais. Em que pese a multiplicidade de fatores que podem ser incluídos em cada um desses níveis, muitas vezes tais possibilidades esbarram na indisponibilidade ou inadequação das informações, especialmente no caso de países em desenvolvimento, como o Brasil.

Tendo em vista as limitações específicas de abarcar, com base nos dados da PNAD 2003, os quatro níveis de agregação mencionados, propõe-se, neste trabalho, considerar dois níveis de determinação: individual e domiciliar/comunitário. O primeiro abarca as características do indivíduo e suas patologias e distúrbios metabólicos relacionados com o diabetes, além de aspectos socioeconômico-culturais, incluindo variáveis como renda, ocupação e escolaridade. O nível domiciliar/comunitário contempla as características comportamentais determinadas pelo ambiente, como dieta alimentar. Definidos estes níveis, é possível analisar como fatores associados, de cunho domiciliar/comunitário, podem exercer influência sobre o evento de interesse, através de fatores individuais, na tentativa de entender os mecanismos por meio dos quais surgem os diferenciais e caminhos através dos quais outras variáveis exercem seu efeito (CRIMMINS; SEEMAN, 2001). 


\section{Métodos}

Os dados utilizados são provenientes da Pesquisa Nacional por Amostra de Domicílios (PNAD) de 2003 (IBGE, 2005). No questionário de 2003, a pergunta sobre morbidade é auto-referida e explicitou a necessidade de um diagnóstico prévio de algum médico ou profissional de saúde.

A amostra correspondeu a 3.692 idosos com 60 anos e mais, residentes em Minas Gerais. Foram excluídos 30 casos com imprecisão em pelo menos uma das variáveis estudadas, resultando em 3.662 pessoas. Trabalhou-se com o universo amostral porque o fator de ponderação somente expande a amostra, mas não interfere no seu desenho.

Os fatores associados foram reunidos em dois níveis de determinação: individual e domiciliar/comunitário. No primeiro, os fatores associados ao diabetes foram agrupados em determinantes mais próximos e próximos e, no segundo, foram reunidos em fatores menos distantes, distantes e mais distantes (MOSLEY; CHEN, 1984).

Não foi possível diferenciar os tipos de diabetes neste trabalho. Contudo, como o do tipo 2 é aproximadamente nove vezes mais prevalente do que o do tipo 1 e normalmente acomete pessoas mais velhas, inferese que os idosos estudados provavelmente apresentam o tipo 2 da doença (OLIVEIRA; MILECH, 2004).

\section{Variáveis}

Os fatores relacionados ao nível individual foram agrupados em: mais próximos, que reúnem características inerentes ao indivíduo (sexo, idade, ter hipertensão e ter doença do coração); e próximos, que englobam aspectos socioeconômicos (instrução, ocupação, renda e raça/cor). No nível domiciliar/comunitário, o número de moradores no domicílio e a relação com a pessoa de referência do domicílio são variáveis classificadas como fatores menos distantes, que expressam a composição intradomiciliar. Entre os fatores distantes, foram agrupadas variáveis que indicam as condições econômicas do domicílio: con- dições gerais e presença de bens. Por fim, os fatores mais distantes contemplaram a situação de residência do indivíduo: situação censitária e região de moradia.

A presença de diabetes foi considerada variável dependente, com resposta que assumiu valores 0 (não) ou 1 (sim).

No nível individual, as variáveis selecionadas foram categorizadas e aquelas com mais de duas categorias foram transformadas em variáveis indicadoras. $\mathrm{Na}$ variável condição de ocupação, foram considerados ocupados os idosos que, no período de referência, estavam trabalhando, os que não exerceram atividade remunerada por motivos como férias, licença e greve e aqueles que procuraram trabalho.

No nível domiciliar/comunitário, foram incluídas variáveis que contemplam diferentes facetas das características domiciliares e do meio. Assim, o domicílio de idoso corresponde àquele no qual o idoso era a pessoa de referência ou cônjuge. As variáveis condições gerais e presença de bens no domicílio indicaram condições socioeconômicas do domicílio e foram consideradas uma proxy de riqueza do domicílio. Ambas foram criadas com base na metodologia de análise de componentes principais (FILMER; PRITCHETT, 2001). Na construção do índice, utilizou-se o pacote estatístico STATA@) (Statistical Data Analysis), versão 8. Quanto às condições gerais do domicílio, o índice obtido condensou dimensões relacionadas às suas condições de saneamento (rede geral de água, rede geral de esgoto, lixo coletado e ter banheiro). Após sua construção, foram criados quartis com base nos escores gerados. Em seguida, os domicílios foram classificados em condições ruins, razoáveis, boas e muito boas. A mesma metodologia foi aplicada utilizando-se um conjunto de variáveis relacionadas à presença de bens no domicílio.

\section{Regressão logística binária multivariada}

Dos 439 idosos que declararam ter diabetes, 417 (95\%) residiam em domicílios independentes e apenas 22 encontravamse em domicílios com presença de outro 
idoso com diabetes. Assim, considerou-se bastante razoável os indivíduos com diabetes serem independentes (não estavam agrupados em domicílios com outros idosos diabéticos - isto poderia evidenciar um efeito de conglomerados de pessoas com diabetes, o que os tornaria dependentes entre si). A opção, então, foi trabalhar com modelos tendo por base a regressão logística binária (uma variável resposta binária - a presença de diabetes) multivariada (múltiplas variáveis independentes). Neste tipo de modelagem, os resultados se aplicam a todos os indivíduos com níveis similares da variável independente na população em estudo. Toda a variação presente entre os indivíduos seria medida por cada uma das variáveis incluídas no estudo efetivamente observadas e medidas. ${ }^{1}$

Cinco modelos são apresentados para a variável-resposta. Se a idéia de fatores associados próximos ao evento é correta e se esses fatores são mensurados sem erro e exaustivamente, outros fatores (mais distantes) não deveriam exercer efeito significativo sobre a variável-resposta, dado que cada fator associado distante deveria atuar sobre a variável-resposta através de fatores próximos (MOSLEY; CHEN, 1984). Logo, a forma de inclusão sucessiva das variáveis no modelo, com base no modelo conceitual, pode sugerir caminhos através dos quais a variável-resposta é afetada, pois se espera que a inclusão sucessiva de fatores próximos influencie os coeficientes de cada fator associado distante, reduzindo sua significância.

Utilizou-se o teste de Wald para verificar a significância individual dos coeficientes. Para avaliar o ajuste geral dos modelos, as razões de verossimilhança foram sucessivamente comparadas.

\section{Resultados}

\section{Características gerais}

Dos 3.662 idosos investigados, 439 $(11,9 \%)$ se auto-referiram diabéticos e 3.223 $(88,1 \%)$ não o fizeram. Entre os diabéticos, $65,8 \%$ eram do sexo feminino. Já a proporção de mulheres entre os não-diabéticos era inferior (53,9\%). As distribuições por idade, entre diabéticos e não-diabéticos, se revelaram bastante similares (Tabela 1).

Entre os idosos sem diabetes, 51,3\% tinham hipertensão, valor que sobe para $73,8 \%$ entre os diabéticos. Os dados revelaram ainda que $17,3 \%$ daqueles sem diabetes apresentaram doença do coração; esta proporção mais do que dobrou entre os diabéticos $(35,8 \%)$.

Quanto às condições socioeconômicas dos idosos com diabetes, verificou-se que a maioria ganhava até um salário mínimo (92,3\%), não estava ocupada $(73,6 \%)$, não era pensionista $(72,9 \%)$, mas se encontrava aposentada $(61,3 \%)$ na semana de referência. Por outro lado, observa-se que, entre estes idosos, ser pensionista, não ser ocupado e não ser aposentado eram situações mais comuns comparativamente àqueles sem diabetes. Além disso, 63,6\% dos idosos diabéticos tinham menos de quatro anos de estudo, enquanto entre aqueles sem diabetes esta proporção era de 59,0\%. Com relação à raça/cor, mais da metade dos idosos diabéticos eram brancos $(52,2 \%)$ e apenas $9,6 \%$ se declararam pretos. A proporção de brancos era levemente inferior, comparativamente aos indivíduos sem diabetes.

Os dados revelaram uma proporção levemente superior de diabéticos residentes em domicílios unipessoais (10,7\%), em rela-

\footnotetext{
${ }^{1}$ Cabe notar que, se houvesse evidência de que os indivíduos estivessem aglomerados em determinados domicílios, seria necessário "controlar" pela perda de independência (covariância entre os idosos no mesmo domicílio). Uma forma seria calcular um desvio para cada domicílio j e para cada indivíduo i, em torno da probabilidade média (populacional) de diabetes (modelo de efeito aleatório). No caso deste estudo, o modelo considerado foi de efeito fixo (HOSMER; LEMESHOW, 2000).
} 
TABELA 1

Distribuição dos idosos com e sem diabetes auto-referido, segundo características selecionadas para o nível individual

Estado de Minas Gerais - 2003

Em porcentagem

\begin{tabular}{|c|c|c|}
\hline Características & $\begin{array}{l}\text { Idosos com diabetes } \\
\text { auto-referido }\end{array}$ & $\begin{array}{c}\text { Idosos sem diabetes } \\
\text { auto-referido }\end{array}$ \\
\hline Total (nos. absolutos) & 439 & 3.223 \\
\hline \multicolumn{3}{|l|}{ Fatores mais próximos } \\
\hline \multicolumn{3}{|l|}{ Sexo } \\
\hline Homens & 34,17 & 46,08 \\
\hline Mulheres & 65,83 & 53,92 \\
\hline \multicolumn{3}{|l|}{ Grupos de idade } \\
\hline 60 a 64 anos & 29,61 & 30,38 \\
\hline 65 a 69 anos & 26,20 & 24,45 \\
\hline 70 a 74 anos & 17,31 & 20,42 \\
\hline 75 a 79 anos & 13,21 & 12,69 \\
\hline 80 a 84 anos & 8,66 & 6,76 \\
\hline 85 anos e mais & 5,01 & 5,31 \\
\hline \multicolumn{3}{|l|}{ Hipertensão } \\
\hline Não tem & 26,20 & 48,74 \\
\hline Tem & 73,80 & 51,26 \\
\hline \multicolumn{3}{|l|}{ Doença do coração } \\
\hline Não tem & 64,24 & 82,69 \\
\hline Tem & 35,76 & 17,31 \\
\hline \multicolumn{3}{|l|}{ Fatores próximos } \\
\hline \multicolumn{3}{|l|}{ Raça/ cor } \\
\hline Branca & 52,16 & 54,61 \\
\hline Preta & 9,57 & 9,74 \\
\hline Parda & 38,27 & 35,65 \\
\hline \multicolumn{3}{|l|}{ Nível de instrução } \\
\hline Sem instrução e menos de 1 ano & 38,04 & 36,83 \\
\hline 1 a 3 anos & 25,51 & 22,12 \\
\hline 4 anos & 22,55 & 22,99 \\
\hline 5 anos ou mais & 13,90 & 18,06 \\
\hline \multicolumn{3}{|l|}{ Renda individual } \\
\hline Até $1 \mathrm{SM}$ & 92,26 & 88,12 \\
\hline Mais de 1 até $2 \mathrm{SM}$ & 3,42 & 5,31 \\
\hline Mais de 2 até $3 \mathrm{SM}$ & 1,37 & 2,39 \\
\hline Mais de 3 até 5 SM & 0,46 & 1,83 \\
\hline Mais de $5 \mathrm{SM}$ & 2,51 & 2,36 \\
\hline \multicolumn{3}{|l|}{ Aposentado } \\
\hline Não & 38,72 & 34,44 \\
\hline Sim & 61,28 & 65,56 \\
\hline \multicolumn{3}{|l|}{ Pensionista } \\
\hline Não & 72,89 & 81,82 \\
\hline Sim & 27,11 & 18,18 \\
\hline \multicolumn{3}{|l|}{ Ocupado } \\
\hline Não & 73,58 & 64,32 \\
\hline Sim & 26,42 & 35,68 \\
\hline
\end{tabular}

Fonte: IBGE. Pesquisa Nacional por Amostra de Domicílios - PNAD 2003.

ção aos não diabéticos (11,7\%). A maioria $(87,7 \%)$ dos idosos com diabetes ocupava a posição de pessoa de referência ou cônjuge no domicílio, sendo esta proporção semeIhante à dos idosos sem diabetes $(87,8 \%)$.

A maioria dos idosos com diabetes $(88,2 \%)$ residia em áreas urbanas, mas somente $32,1 \%$ na Região Metropolitana de Belo Horizonte. Já para os não-diabéticos, essas proporções eram um pouco menores: $82,8 \%$ e $28,8 \%$, respectivamente. Quanto às variáveis relacionadas às condições específicas do domicílio, as distribuições foram semelhantes (Tabela 2). 
TABELA 2

Distribuição dos idosos com e sem diabetes auto-referido, segundo características selecionadas para o nível domiciliar/comunitário

Estado de Minas Gerais - 2003

Em porcentagem

\begin{tabular}{|c|c|c|}
\hline Características & $\begin{array}{l}\text { Idosos com diabetes } \\
\text { auto-referido }\end{array}$ & $\begin{array}{c}\text { Idosos sem diabetes } \\
\text { auto-referido }\end{array}$ \\
\hline Total (nos. absolutos) & 439 & 3.223 \\
\hline \multicolumn{3}{|c|}{ Fatores menos distantes } \\
\hline \multicolumn{3}{|c|}{ No. de moradores no domicílio } \\
\hline 1 & 10,71 & 11,73 \\
\hline 2 & 32,80 & 29,10 \\
\hline 3 & 20,96 & 22,93 \\
\hline 4 & 35,54 & 36,24 \\
\hline \multicolumn{3}{|c|}{ Se o idoso é pessoa de referência ou cônjuge } \\
\hline Não & 12,30 & 12,22 \\
\hline Sim & 87,70 & 87,78 \\
\hline \multicolumn{3}{|c|}{ Fatores distantes } \\
\hline \multicolumn{3}{|c|}{ Condições gerais do domicílio } \\
\hline Ruins & 22,10 & 25,57 \\
\hline Razoáveis & 24,83 & 25,50 \\
\hline Boas & 27,11 & 24,08 \\
\hline Muito boas & 25,97 & 24,85 \\
\hline \multicolumn{3}{|c|}{ Presença de bens no domicílio } \\
\hline Muito pouco & 36,90 & 37,36 \\
\hline Pouco & 20,50 & 20,14 \\
\hline Razoável & 21,87 & 21,78 \\
\hline Bastante & 20,73 & 20,73 \\
\hline \multicolumn{3}{|l|}{ Fatores mais distantes } \\
\hline \multicolumn{3}{|l|}{ Residência na $R M B H$} \\
\hline Não & 67,88 & 71,21 \\
\hline Sim & 32,12 & 28,79 \\
\hline \multicolumn{3}{|l|}{ Situação censitária } \\
\hline Rural & 11,85 & 17,19 \\
\hline Urbana & 88,15 & 82,81 \\
\hline
\end{tabular}

Fonte: IBGE. Pesquisa Nacional por Amostra de Domicílios - PNAD 2003.

\section{Análise multivariada}

A estratégia adotada consistiu em estimar modelos com as variáveis de natureza domiciliar/comunitária (modelos 1, 2 e 3) e, depois, agregar modelos com as variáveis individuais (modelos 4 e 5). Foram incluídos, no modelo 1, os fatores mais distantes (região de residência e situação censitária), no modelo 2, os distantes (condições gerais do domicílio e presença de bens no domicílio) e, no modelo 3 , os menos distantes (número de moradores no domicílio e domicílio de idoso). Em seguida, no nível individual, o modelo 4 contemplou o acréscimo dos fatores próximos (raça/ cor, nível de instrução, renda individual, aposentado, pensionista e ocupação) e, no modelo 5 , foram aduzidos os fatores mais próximos (sexo, idade, hipertensão e doença do coração).

No modelo 1, a região de residência não apresentou variação estatisticamente significativa com a prevalência de diabetes entre idosos, relação esta que permaneceu em todos os modelos. Morar na RMBH não resultou em efeito significativo sobre a prevalência de diabetes, mas a residência em área urbana aumentou a chance de diabetes em 50,5\%, em comparação com idosos da área rural.

A introdução, no modelo 2, dos fatores distantes mostrou que as condições gerais e a presença de bens no domicílio não afetaram de maneira estatisticamente significativa a chance de o idoso ter diabetes. Esta situação se manteve nos demais modelos. Contudo, a residência na área urbana au- 
mentou a chance de ter diabetes em $69,8 \%$ (modelo 2 em relação ao modelo 1).

O acréscimo, no modelo 3 , dos fatores menos distantes reforçou o efeito de residir em área urbana. Já o número de idosos moradores na residência e ser a pessoa de referência no domicílio ou cônjuge não determinaram variação significativa na chance de ter diabetes.

No modelo 4 foram incluídos fatores próximos, do nível individual. A chance de idosos urbanos terem diabetes diminuiu de $73,0 \%$, no modelo 3 , para $56,2 \%$, no 4 , indicando que as variáveis individuais acrescidas "roubaram" parte do efeito de situação censitária. Raça/cor não apresentou variação estatisticamente significativa no que se refere à chance de o idoso ter diabetes. Contudo, quando comparados aos idosos com cinco ou mais anos de estudo, aqueles pertencentes às outras categorias de nível de instrução apresentaram maior chance de ter diabetes.

A chance de idosos residentes em domicílios unipessoais terem diabetes em relação àquela dos que viviam em domicílios com dois moradores resultou significativamente inferior (modelos 3, 4 e 5). No modelo 3 esse indicador não se revelou estatisticamente significativo e, à medida que as variáveis do indivíduo foram sendo incluídas no modelo, a significância de apenas um morador aumentou, embora tenha havido redução no valor do coeficiente. Assim, conforme fatores mais próximos foram considerados no modelo, as diferenças entre domicílios unipessoais em comparação àqueles de dois moradores aumentaram.

$\mathrm{Na}$ presença de outros fatores associados ao diabetes, o fato de o idoso estar aposentado não reduziu a chance de diabetes. Contudo, ser pensionista e não estar ocupado na semana de referência apareceram como fatores agravantes, comparativamente às categorias de referência.

Quanto à renda individual, mesmo no modelo final, entre idosos com renda de mais de 3 até 5 salários mínimos, a chance de ter diabetes revelou-se $77,7 \%$ menor do que para aqueles com renda superior a cinco SM.
Ao serem inseridos os fatores mais próximos (modelo 5), manteve-se a chance inferior de idosos com renda de mais de 3 até $5 \mathrm{SM}$ terem diabetes, relativamente àqueles com renda superior a cinco SM. Também os idosos com renda de até um SM passaram a ter uma chance de ter diabetes menor $(45,7 \%)$, relativamente àqueles no estrato de renda mais elevada. A inclusão dos fatores individuais mais próximos retirou a significância estatística do efeito da condição de ocupado sobre a prevalência de diabetes. Quanto ao sexo, no modelo final, a chance de ter diabetes resultou $31,8 \%$ maior entre as idosas, relativamente aos idosos. Ter hipertensão ou doença do coração mais do que duplicou a chance de o idoso ter diabetes, em comparação aos não portadores dessas doenças. Verificou-se ainda que a chance de ter diabetes era $26,8 \%$ inferior para os de 70 a 74 anos, comparativamente àqueles com 60 a 64 anos.

Finalmente, o teste da razão de verossimilhança revelou que o modelo 2 não obteve ajuste significativamente superior ao modelo 1 (valor de $p=0,7791$ ). O modelo 3 também não se apresentou significativamente superior ao 2 (valor de $p=0,4734$ ). Já o modelo 4 mostrou-se superior ao 3 (valor de $p<$ 0,001 ) e o modelo 5 foi superior ao 4 (valor de $p<0,001)$.

\section{Discussão}

É importante destacar que pode haver subestimações ou superestimações na mensuração da prevalência de doenças crônicas, com base na PNAD 2003, sendo esta uma limitação do estudo. É possível que haja falha de memória, especialmente entre os idosos, além da ausência de diagnóstico nos casos em que não tenha havido procura por atendimento (por falta ou dificuldade de acesso, ou porque não houve percepção de sintomas da doença). Além disso, nem sempre a informação foi transmitida pela pessoa de referência (30,6\% das respostas foram fornecidas por outro morador do domicílio e 2,8\% por um não-morador). Contudo, em $2 / 3$ dos casos a informação foi dada pela própria pessoa e muitos estudos são baseados em 
informações auto-referidas, apresentando resultados coerentes.

Entre os resultados, sobressai o efeito de renda individual do idoso sobre variações na prevalência de diabetes. Mesmo controlando-se pelos demais fatores, a chance de idosos com renda de até um SM e com renda de mais de 3 até 5 SM terem diabetes é menor do que aquela de idosos na categoria de renda mais elevada (mais de cinco $\mathrm{SM}$ ). Este resultado está em consonância com outros estudos (BROWN et al., 2004; MCEWEN et al., 2007). É possível que a renda individual influencie fortemente o acesso a serviços de saúde, que, por sua vez, pode influenciar o nível de percepção e procura por atendimento, afetando a auto-referência da doença. Diante disso, se tivesse sido mortalidade a variável-resposta, esperarse-ia um efeito indireto da renda individual do idoso. Porém, no caso da prevalência auto-referida de morbidade, com base em diagnóstico médico ou de outro profissional de saúde, a relação direta encontrada não chega a ser inesperada. Na verdade, maior acesso a serviços de saúde, assim como as demais vantagens inerentes a idosos situados na categoria de maior renda individual, pode significar, também, chance maior de ter a doença diagnosticada e, portanto, de auto-referi-la em maior proporção, em comparação aos idosos de menor renda.

Destacam-se a forte associação entre prevalência de diabetes em idosos e a presença de hipertensão e doença do coração, assim como com a residência em área urbana, o sexo feminino, a condição de pensionista e o grau de instrução inferior a quatro anos. Estes fatores contribuíram aumentando a chance de prevalência de diabetes. Quanto à condição de pensionista, é possível que indivíduos que recebam este tipo de benefício, por estarem inseridos em contexto socioeconômico beneficiado, tenham chance mais elevada de prevalência de diabetes. Contudo, se renda fosse a mediadora da associação entre prevalência de diabetes e ser pensionista, o efeito desta variável desapareceria na presença de renda do idoso. Este resultado não é intuitivo e demanda novos estudos. Já no caso de nível de instrução, o resultado era esperado e corrobora estudos que encontraram associação entre conhecimento sobre o diabetes e suas complicações, o qual é associado à incidência e à prevalência de diabetes (MURUGESAN et al., 2007). Destacam-se, por outro lado, como fatores protetores, a residência do idoso em domicílio unipessoal e idades entre 70 e 74 anos. Este resultado possivelmente revela um viés de sobrevivência seletiva. Assim, o achado para a população de 70 a 74 anos e também para a mais idosa (ausência de maior chance de diabetes comparativamente às idades $60 \mathrm{a}$ 64) não surpreende e corrobora estudos anteriores que verificaram o estreitamento dos diferenciais de saúde em idades avançadas (BECKETT, 2000). Seria razoável supor que, neste estudo, indivíduos com menos de 70 anos teriam uma desvantagem em relação a um grupo selecionado mais robusto, de 70 anos e mais.

Esperava-se que fatores mais distantes, que estavam relacionados ao nível domiciliar/comunitário, tivessem sua significância atenuada pela inclusão de fatores mais próximos, ligados ao nível individual, mas isso não ocorreu para algumas variáveis importantes. Mesmo controlando pelos indicadores sociodemográficos e de saúde do indivíduo, a situação de residência e o arranjo intradomiciliar mantiveram sua significância estatística. Provavelmente, o efeito da área de residência é explicado por características de estilo de vida, não controladas pelo modelo. Estudos têm revelado que o aumento se deve aos novos padrões culturais, tais como alteração de hábitos alimentares e redução de atividade física, adquiridos com o estilo de vida urbano (OLIVEIRA, 2004; LIMA-COSTA et al., 2004). De fato, uma das conseqüências da mudança ambiental é a obesidade, a forma mais comum de resistência à ação da insulina, associada também com a hipertensão (OLIVEIRA, 2004).

A associação encontrada entre composição intradomiciliar e diabetes só se revelou com significância estatística quando foram adicionadas as características socioeconômicas do indivíduo (renda, instrução, raça/ cor, ocupação, aposentadoria, pensão), mantendo essa significância mesmo após 
TABELA 3

Razões de chance dos fatores associados ao diabetes entre idosos, segundo características selecionadas para os níveis individual e domiciliar/comunitário

Estado de Minas Gerais - 2003

\begin{tabular}{|c|c|c|c|c|c|}
\hline Características & Modelo 1 & Modelo 2 & Modelo 3 & Modelo 4 & Modelo 5 \\
\hline \multicolumn{6}{|l|}{ Fatores mais distantes } \\
\hline \multicolumn{6}{|l|}{ Reside na RMBH } \\
\hline Não & 1,00 & 1,00 & 1,00 & 1,00 & 1,00 \\
\hline Sim & 1,09 & 1,13 & 1,14 & 1,12 & 1,14 \\
\hline \multicolumn{6}{|l|}{ Situação censitária } \\
\hline Rural & 1,00 & 1,00 & 1,00 & 1,00 & 1,00 \\
\hline Urbana & $1,51(3)$ & $1,70(3)$ & $1,73(3)$ & $1,56(2)$ & $1,61(2)$ \\
\hline \multicolumn{6}{|l|}{ Fatores distantes } \\
\hline \multicolumn{6}{|l|}{ Condições gerais do domicílio } \\
\hline Ruins & & 1,07 & 1,08 & 1,033 & 1,04 \\
\hline Razoáveis & & 0,91 & 0,91 & 0,862 & 0,85 \\
\hline Boas & & 1,10 & 1,10 & 1,088 & 1,08 \\
\hline Muito boas & & 1,00 & 1,00 & 1,00 & 1,00 \\
\hline \multicolumn{6}{|l|}{ Presença de bens no domicílio } \\
\hline Muito pouco & & 1,23 & 1,24 & 1,00 & 1,05 \\
\hline Pouco & & 1,08 & 1,08 & 0,90 & 0,94 \\
\hline Razoável & & 1,09 & 1,09 & 0,97 & 1,01 \\
\hline Bastante & & 1,00 & 1,00 & 1,00 & 1,00 \\
\hline \multicolumn{6}{|l|}{$\begin{array}{l}\text { Fatores menos distantes } \\
\text { № de moradores no domicílio }\end{array}$} \\
\hline 1 & & & 0,77 & $0,70(2)$ & $0,68(2)$ \\
\hline 2 & & & 1,00 & 1,00 & 1,00 \\
\hline 3 & & & 0,81 & 0,80 & 0,82 \\
\hline 4 & & & 0,86 & 0,84 & 0,84 \\
\hline \multicolumn{6}{|c|}{ Idoso é a pessoa de referência no domicílio } \\
\hline Não & & & 1,00 & 1,00 & 1,00 \\
\hline Sim & & & 1,00 & 1,18 & 1,20 \\
\hline \multicolumn{6}{|l|}{ Fatores próximos } \\
\hline \multicolumn{6}{|l|}{ Raça/ cor } \\
\hline Branca & & & & 1,00 & 1,00 \\
\hline Preta & & & & 1,02 & 0,93 \\
\hline Parda & & & & 1,11 & 1,14 \\
\hline \multicolumn{6}{|l|}{ Nível de instrução } \\
\hline Sem instruçẩo e menos de 1 ano & & & & $1,55(2)$ & $1,37(1)$ \\
\hline 1 a 3 anos & & & & $1,73(3)$ & $1,54(2)$ \\
\hline 4 anos & & & & $1,37(1)$ & 1,26 \\
\hline 5 anos ou mais & & & & 1,00 & 1,00 \\
\hline Renda individual & & & & & \\
\hline Até $1 \mathrm{SM}$ & & & & 0,63 & $0,54(1)$ \\
\hline Mais de 1 até 2 SM & & & & 0,52 & 0,52 \\
\hline Mais de 2 até 3 SM & & & & 0,50 & 0,52 \\
\hline Mais de 3 até 5 SM & & & & $0,22(1)$ & $0,23(1)$ \\
\hline Mais de 5 SM & & & & 1,00 & 1,00 \\
\hline Aposentado & & & & & \\
\hline Não & & & & 1,00 & 1,00 \\
\hline Sim & & & & 1,00 & 1,14 \\
\hline Pensionista & & & & & \\
\hline Não & & & & 1,00 & 1,00 \\
\hline Sim & & & & $1,58(4)$ & $1,47(3)$ \\
\hline Ocupado & & & & & \\
\hline Nẫo & & & & 1,00 & 1,00 \\
\hline Sim & & & & $0,76(2)$ & 0,86 \\
\hline Fatores mais próximos & & & & & \\
\hline Sexo & & & & & \\
\hline Homens & & & & & 1,00 \\
\hline Mulheres & & & & & $1,32(2)$ \\
\hline Grupos de idade & & & & & \\
\hline 60 a 64 anos & & & & & 1,00 \\
\hline 65 a 69 anos & & & & & 1,06 \\
\hline 70 a 74 anos & & & & & $0,73(1)$ \\
\hline 75 a 79 anos & & & & & 0,85 \\
\hline 80 a 84 anos & & & & & 1,10 \\
\hline 85 e mais anos & & & & & 0,82 \\
\hline Hipertensão & & & & & \\
\hline Não & & & & & 1,00 \\
\hline Sim & & & & & $2,12(3)$ \\
\hline Doença do coração & & & & & \\
\hline Não & & & & & 1,00 \\
\hline Sim & & & & & $2,16(3)$ \\
\hline Número de casos & 3.662 & 3.662 & 3.662 & 3.662 & 3.662 \\
\hline Log Verossimilhanca & -1338.2 & -1336.6 & -1334.9 & -1314.3 & -1253.4 \\
\hline
\end{tabular}

Fonte: IBGE. Pesquisa Nacional por Amostra de Domicílios - PNAD 2003.
(1) Valor de $p<0,1$.
(3) Valor de $p<0,01$.
(2) Valor de $p<0,05$
(4) Valor de $p<0,001$. 
a inclusão dos fatores mais próximos (sexo, idade, instrução, hipertensão, doença do coração). Assim, residir em domicílio unipessoal se revelou um fator protetor em relação à prevalência de diabetes, comparativamente aos domicílios com duas pessoas, resultado este inesperado. De fato, no caso da composição domiciliar, o que se tem é que, para atender às demandas de dietas especiais e acompanhamento sistemático do idoso aos exames e consultas, é necessário que outras pessoas no domicílio se encarreguem dessas atividades. Era de se esperar uma chance maior de prevalência de diabetes entre aqueles idosos que moravam sozinhos, por, possivelmente, apresentarem uma dieta alimentar pouco nutritiva e pouco balanceada, como função direta de estar só. Além disso, pode-se cogitar a idéia de que essas pessoas, por viverem só, teriam maiores tendências depressivas e pouco investimento na qualidade de suas vidas. Contudo, não se sabe se a composição intradomiciliar do idoso com diabetes se deu pré ou pós-diagnóstico da doença, o que é um viés de causalidade reversa inerente a estudos seccionais. Ademais, deve-se considerar que essa variável passa a ter efeito estatisticamente significativo com a inserção de condições socioeconômicas do idoso, o que talvez revele que esse idoso que mora só possa ter melhores condições no que se refere tanto ao acesso aos serviços de saúde quanto ao autocuidado.

A chance de ter diabetes revelou-se maior entre as mulheres, em relação aos homens, independentemente do controle pelas demais variáveis consideradas no estudo. Mesmo levando em conta que a

\section{Referências bibliográficas}

BECKETT, M. Converging health inequalities in later life-an artifact of mortality selection? Journal of Health and Social Behavior, North Carolina, 41(1): 106-119, 2000.

$\mathrm{BLOCH}, \mathrm{K}$. V. Fatores de risco cardiovasculares e para o diabetes mellitus. In: LESSA, I. O. (Org.). $\mathbf{O}$ adulto brasileiro e as doenças da modernidade: epidemiologia das doenças crônicas não-transmissíveis. São Paulo; Editora Hucitec, 1998, p. 43-72. variável-resposta é auto-referida e o fato de o acesso aos serviços de saúde ser maior na população feminina, acredita-se que as transformações hormonais, experimentadas pós-menopausa, favoreçam a deposição de gordura, que consiste em preditor do diabetes (SCHNEIDER, 2006). Adicionalmente, é razoável supor que as coortes de mulheres idosas, na sua socialização de gênero, não foram treinadas para atividades físicas que, praticadas ao longo da vida, contribuem para menor incidência de doenças crônicas, como o diabetes (MONTEIRO et al., 2004; SALLES-COSTA et al., 2003).

A relevância da análise de aspectos sociodemográficos dos idosos com diabetes mellitus auto-referido, apesar das limitações desse tipo de variável, está na compreensão de todo esse processo e na possibilidade de subsidiar delineamentos de programas de política pública que visam minorar o ônus advindo da prevalência dessa doença, favorecendo, do ponto de vista individual, uma vida com maior qualidade. Já do ponto de vista geracional, o diagnóstico precoce, associado ao tratamento adequado, pode evitar ou, pelo menos, postergar o aparecimento de complicações e reduzir seu impacto sobre as variáveis demográficas. Do ponto de vista de saúde pública, resta mencionar a redução dos custos gerados pela carga dessa doença sobre o sistema de saúde. Os resultados apresentados apontam para esses e novos desafios, e ainda fornecem elementos para reflexão acerca das ações passíveis de serem implementadas, com base no conhecimento já adquirido sobre fatores associados à prevalência do diabetes entre a população idosa.

BROWN, A. F. et al. Socioeconomic position and health among persons with diabetes mellitus: a conceptual framework and review of the literature. Epidemiologic Reviews, Baltimore, 26: 63-77, 2004.

CRIMMINS, E. M.; SEEMAN, T. E. Integrating biology into demographic research on health and aging (with a focus on the MacArthur Study of Successful Aging). In: FINCH, C. E.; VAUPEL, J. W. (Eds). Cells and surveys: 
should biological measures be included in social science research. National Academy Press, 2001, p. 9-41.

FILMER, D.; PRITCHETT, L. H. Estimation wealth effects without expenditure data - or tears: an application of educational enrollment in the state of India. Demography, United States, 38: 115-32, 2001.

IBGE - Instituto Brasileiro de Geografia e Estatística. Pesquisa nacional por amostra de domicílios: acesso e utilização de serviços de saúde: 2003. Rio de Janeiro: IBGE, 2005.

LEITE, I. C.; BELTRÃO, K. I.; RODRIGUES, R. N.; VALENTE, J. G.; CAMPOS, M. R.; SCHRAMM, J. M. A. Projeção da carga de doença no Brasil (1998-2013). In: BUSS, P. M.; TEMPORÃO, J. G.; CARVALHEIRO, J. R. Vacinas, soros e imunizações no Brasil. Rio de Janeiro: Fiocruz, 2005, p.51-65.

LIMA-COSTA, M. F.; PEIXOTO, S.V.; GIATTI, $L$. Tendências da mortalidade entre idosos brasileiros (1980-2000). Epidemiologia e serviços de saúde, Brasília, 13(4), p.217228, 2004.

HOSMER, D. W.; LEMESHOW, S. Applied logistic regression. New York: John Wiley \& Sons Inc., 2000.

LOURENÇO, R. A. Diabetes no idoso. In: OLIVEIRA, J. E. P.; MILLECH, A. Diabetes mellitus: clínica diagnóstico, tratamento interdisciplinar. São Paulo: Editora Atheneu, 2004, p. 339-344.

MCEWEN, L. N. et. al. Risk factors for mortality among patients with diabetes: the Translating Research Into Action for Diabetes (TRIAD) Study. Diabetes Care, United States, 30(7):1736-41, 2007.

MONTEIRO C. A. et. al. A descriptive epidemiology of leisure-time physical activity in Brazil, 1996-1997. Rev Panam Salud Publica, Washington, 14(4): 246-54, 2003.

MOSLEY, W. H.; CHEN, L. C. An analytical framework for the study of child survival in developing countries. Population and Development Review, New York; 10 (Suppl): 25-45, 1984.

MURUGESAN, N. et al. Awareness about diabetes and its complications in the general and diabetic population in a city in southern India. Diabetes Research and Clinical Practice, Oxford, 77(3): 433-437, 2007.

OLIVEIRA, J. E. P. Conceitos, classificação e diagnóstico do diabetes mellitus. In: OLIVEIRA, J. E. P.; MILLECH, A. Diabetes mellitus: clínica diagnóstico, tratamento interdisciplinar. São Paulo: Editora Atheneu, 2004, p. 7-18.

OLIVEIRA, J. E. P.; MILECH, A. Diabetes: passado, presente e futuro. In: OLIVEIRA, J. E. P.; MILLECH, A. Diabetes mellitus: clínica diagnóstico, tratamento interdisciplinar. São Paulo: Editora Atheneu, 2004, p. 1-6.

SALLES-COSTA R. et. al. Gênero e prática de atividade física de lazer. Cad. Saúde Pública, Rio de Janeiro, 19 (supl.2): 325333, 2003.

SCHNEIDER, J. G.; TOMPKINS, C.; BLUMENTHAL, R. S.; MORA, S. The metabolic syndrome in women. Cardiology in Review, Boston, 14(6): 286-91, 2006.

WHO - World Health Organization. Screening for type 2 diabetes: report of a World Health Organization and International Diabetes Federation meeting. Geneva: Department of Noncommunicable disease surveillance, 2003. Disponível em: <http://www.who.int/ diabetes/currentpublications/en/>. Acesso em: 09 dez. 2005.

\section{Resumen}

Factores asociados a la prevalencia de diabetes auto-referida entre ancianos de Minas Gerais, 2003

La prevalencia de diabetes viene aumentando mundialmente, y en este artículo, fueron analizados algunos de sus factores asociados, focalizando el segmento de ancianos residentes 
en Minas Gerais. Fue utilizado el método de estudio seccional, con datos de la PNAD-2003, abarcando una muestra de 3.662 personas con 60 años y más. Los factores asociados fueron organizados según los niveles individuales y domiciliarios / comunitarios, y analizados por medio de cinco modelos de regresión logística multivariada. El primer modelo incluyó solamente variables más distantes; las demás fueron incluidas sucesivamente, de acuerdo con su proximidad a la diabetes. En el modelo final, la probabilidad de tener diabetes fue mayor para: los ancianos residentes en el área urbana en relación a la rural; las mujeres versus los hombres; los hipertensos en relación a los no hipertensos; aquéllos con enfermedades del corazón en relación a los sin enfermedades del corazón; pensionistas versus no pensionistas; sin instrucción y con 1 a 3 años de estudios en comparación a los con cinco años o más de estudios. La probabilidad fue menor entre aquéllos en hogares unipersonales versus los de más de una persona, con 70 a 74 años versus 60 a 64 años, con ingresos inferiores a un salario mínimo y más de 3 hasta 5 salarios mínimos en relación a aquéllos con cinco y más salarios mínimos. La relación entre el ingreso individual del anciano y la diabetes indica la necesidad de estudios que investiguen el acceso de los ancianos a los servicios de salud y que influencian la auto-referencia a la morbilidad.

Palabras-clave: Factores asociados. Diabetes. Diabetes mellitus. Diabetes mellitus tipo 2.

\section{Abstract}

Factors associated with the prevalence of diabetes as self-reported by elderly persons in the State of Minas Gerais, Brazil, 2003

The prevalence of diabetes has been increasing worldwide. This study has the objective of analyzing the prevalence of self-reported diabetes among the elderly of age 60 or over living in the State of Minas Gerais, Brazil. Methods: The study is based on cross-sectional data derived from PNAD-2003 taken from a sample of 3662 persons aged 60 years or over. Factors associated with diabetes were organized according to individual and community/household levels and analyzed using five multivariate logistic regression models. The first model included only more distant variables while the others were included progressively, according to their proximity to diabetes. In the final model, the chance of acquiring diabetes was greater for elderly residents living in urban areas over rural areas, women over men, those who have high blood pressure over those who do not, those with heart disease over those without, pension receivers over non-receivers, and those without instruction, or from one to three years of schooling over those with five or more years of study. The chances of having diabetes was greater for those in single-person households versus those with more than one person, those between ages 70 and 74 over those between 60 and 64, as well as those with income below one minimum wage and between three and five minimum wages over those with incomes of five times the minimum wage or over. Conclusion: The relationship between individual income and diabetes indicates that more research is needed to investigate the association between the access to health services by the elderly and self-reported diabetes, even when other demographic and socioeconomic factors are controlled.

Keywords: Associated factors. Diabetes. Diabetes mellitus.

Recebido para publicação em 28/04/2008. Aceito para publicação em 14/11/2008. 